\title{
High Energy Resolvent Estimates for Generalized Many-Body Schrödinger Operators
}

\author{
By
}

Arne JENSEN*

\begin{abstract}
We prove high energy resolvent estimates for a class of generalized many-body Schrödinger operators. The proofs are based on an extension of Mourre's commutator method.
\end{abstract}

\section{$\S 1$. Introduction}

Let $X=\boldsymbol{R}^{m}$ and let $H_{0}=-\Delta$ on $\mathscr{D}\left(H_{0}\right)=H^{2}(X)$, the usual Sobolev space of order 2. Let $\pi_{j}, j=1,2, \cdots, N$, be orthogonal projections in $X$. Let $X_{j}=\pi_{j}(X)$ be the range of $\pi_{j}$. We consider potentials which are real-valued functions defined on $X_{j}$. Let $x_{j}$ be the coordinates on $X_{j}$, and let $\nabla_{j}$ denote the corresponding gradient. For an integer $n \geqq 1$ we introduce the following

Assumption 1.1. Let $V_{j} \in C^{n}\left(X_{\jmath}\right), j=1, \cdots, N$, such that

$$
\sup _{x_{j} \in X_{j}} \sum_{l=0}^{n}\left|\left(\left(x_{j} \cdot \nabla_{j}\right)^{l} V_{j}\right)\left(x_{j}\right)\right|<\infty .
$$

We will introduce coupling constants $g_{j}>0, j=1, \cdots, N$, and define for $g=$ $\left(g_{1}, \cdots, g_{N}\right) \in \boldsymbol{R}_{+}^{v}$

$$
V(g)=\sum_{j=1}^{N} g_{j} V_{j}\left(\pi_{\jmath}(x)\right) .
$$

Let $H=H_{0}+V(g)$. If we impose the additional condition $V_{j}\left(x_{j}\right) \rightarrow 0$ as $\left|x_{j}\right|$ $\rightarrow \infty$, then $H$ is a generalized many-body Schrödinger operator of the type considered in $[2,4]$. Under this additional condition the structure of the spectrum is investigated by these authors. We do not need the decay condition here.

Let $R(z ; V(g))=(H-z)^{-1}$ denote the resolvent. Let $\langle x\rangle=\left(1+x^{2}\right)^{1 / 2}$ and let for $s \in \boldsymbol{R}$ the weighted $L^{2}$-space be given by

$$
L^{2, s}(X)=\left\{f \in L_{\mathrm{loc}}^{2}(X) \mid\|f\|_{s}=\left\|\langle x\rangle^{s} f\right\|_{L^{2}}<\infty\right\} .
$$

The bounded operators from $L^{s}(X)$ to $L^{s^{\prime}}(X)$ are denoted by $\mathscr{B}\left(s, s^{\prime}\right)$. Let $V_{\text {J }}$

Communicated by H. Araki, July 26, 1988.

* Matematisk Institut, Aarhus Universitet, DK-8000 Aarhus C, Denmark. 
satisfy Assumption 1.1, $n \geqq 1$, and introduce

$$
\mu\left(V_{j}\right)=\max \left\{0, \sup _{x_{j} \in X_{j}}\left(V_{j}\left(x_{j}\right)+\frac{1}{2} x_{j} \cdot \nabla_{j} V_{j}\left(x_{j}\right)\right)\right\} 。
$$

A special case of the main results can be stated as follows. We note that it is only the high energy estimate (1.4) which is new. The other results have been obtained in $[10,7]$.

Theorem 1.2. Let $V_{j}$ satisfy Assumption 1.1 for some $n \geqq 2$. There exists $\lambda_{0}>0$ such that for all $\lambda \geqq \lambda_{0}$ and all $g \in \boldsymbol{R}_{+}^{N}$ satisfying $\lambda \geqq c_{0} \max \left\{g_{j}\right\}$ and $\lambda \geqq \delta$ $\cdot \sum_{j=1}^{N} g_{j} \mu\left(V_{j}\right)$ for some $c_{0}>0$ and $\delta>1$ the following results hold:

(i) Let $s>1 / 2$. Then the boundary values

$$
R(\lambda \pm i 0 ; V(g))=\lim _{\varepsilon \downarrow 0} R(\lambda \pm i \varepsilon ; V(g))
$$

exist in $\mathscr{B}(s,-s)$ in operator norm.

(ii) For $k=0,1, \cdots, n-2, s>k+(1 / 2)$ the map $\lambda \mapsto R(\lambda \pm i 0 ; V(g))$ is $k$ times continuously differentiable from $\left(\lambda_{0}, \infty\right)$ to $\mathcal{B}(s,-s)$. The following estimate holds:

$$
\left\|\left(\frac{d}{d \lambda}\right)^{k} R(\lambda \pm i 0 ; V(g))\right\|_{\mathscr{B}(s,-s)} \leqq C \lambda^{-(k+1) / 2} .
$$

The result (1.4) was obtained for $k=0$ with a large coupling constant $g$ in [19] under Assumption 1.1 with $n=2$. High energy estimates for various $k$ with fixed coupling constant was obtained for two-body Schrödinger operators in $[16,1,9,6]$, and for general elliptic operators in $[12,13]$. In $[3]$ high energy resolvent estimates for two-body Schrödinger operators were obtained using a variant of Mourre's technique with a modified conjugate operator.

In this paper we combine the technique developed in [7] with the scaling argument from [19]. The main idea is the observation that the Mourre method for proving a priori resolvent estimates is constructive in the sense that constants in the estimates can be controlled explicitly, or parameter dependence can be followed precisely. See $[5,8,15,19]$ for other results using this observation.

The contents of the paper can briefly be described as follows: In Section 2 we state the precise resolvent estimates obtained using localizations based on the generator of dilations. In Section 3 we give the proofs. In Section 4 we prove Theorem 1.2 and related results. In Section 5 we give some semiclassical resolvent estimates, which are easy consequences of our other results. Section 6 contains a different approach to constructive resolvent estimates for Schrödinger operators with potentials that are homogeneous functions. Section 7 contains some remarks.

\section{§2. Statement of Results}

Let $A=1 / 2 i(x \cdot \nabla+\nabla \cdot x)$ denote the generator of dilations in $L^{2}(X)$. We use 
the following localizing operators :

$$
\rho_{a}=\left(a^{2}+A^{2}\right)^{-1 / 2}, \quad a \geqq 1
$$

$P_{A}^{+}$the spectral projection for $(0, \infty)$

$P_{A}^{-}$the spectral projection for $(-\infty, 0)$.

Let $V_{j}$ satisfy Assumption 1.1 for some $n \geqq 1$. Throughout this paper we use the following condition for all $\lambda>0$ and $g \in \boldsymbol{R}_{+}^{N}$ :

$$
\begin{aligned}
& \lambda \geqq \delta \sum_{j=1}^{N} g_{j} \mu\left(V_{j}\right), \quad \delta>1, \\
& \lambda \geqq c_{0} \max \left\{g_{\jmath} \mid j=1, \cdots, N\right\}, \quad c_{0}>0 .
\end{aligned}
$$

The constants $\delta$ and $c_{0}$ are assumed fixed throughout the paper. The dependence of various constants in estimates on $\delta$ and $c_{0}$ will not be mentioned explicitly.

For two-body Schrödinger operators (i.e. $N=1$ and $X_{1}=X$ ) the condition (2.4) implies the non-trapping condition on the corresponding classical system, see e.g. the comments in [15].

Theorem 2.1. Let $V_{j}$ satisfy Assumption 1.1, $n \geqq 2$. Then there exists $\lambda_{0}>0$ such that for all $\lambda \geqq \lambda_{0}$ and all $g \in \boldsymbol{R}_{+}^{N}$ satisfying (2.4) the following results hold:

(i) For $l=1, \cdots, n-1$ let $s_{1}, s_{2}$ satisfy $s_{1}>l-1 / 2, s_{2}>l-1 / 2$. There exists $c_{n}=c_{n}\left(s_{1}, s_{2}\right)>0$ such that for all $a \geqq 1$ and all $\varepsilon>0$

$$
\left(a_{l}\right) \quad\left\|\rho_{a}^{s_{1}} R(\lambda+i \varepsilon ; V(g))^{l} \rho_{a}^{s_{2}}\right\| \leqq c_{n} \lambda^{-l} a^{l-s_{1}-s_{2}} .
$$

(ii) The boundary value

$$
\lim _{\varepsilon \downarrow 0} \rho_{a}^{s_{1}} R(\lambda+i \varepsilon ; V(g)) \rho_{a}^{s_{2}} \equiv \rho_{a}^{s_{1}} R(\lambda+i 0 ; V(g)) \rho_{a}^{s_{2}}
$$

exists in operator norm. It is $l-1$ times continuously differentiable with a Hölder continuous $l-1^{\text {st }}$ derivative which satisfies the estimate

$$
\left\|\left(\frac{d}{d \lambda}\right)^{l-1} \rho_{a}^{s_{1}} R(\lambda+i 0 ; V(g)) \rho_{a}^{s_{2}}\right\| \leqq c_{n}(l-1) ! \lambda^{-l} a^{l-s_{1}-s_{2}} .
$$

Theorem 2.2. Let $V_{j}$ satisfy Assumption 1.1, $n \geqq 2$. Then there exists $\lambda_{0}>0$ such that for all $\lambda \geqq \lambda_{0}$ and all $g \in \boldsymbol{R}_{+}^{N}$ satisfying (2.4) the following results hold:

(i) For $l=1, \cdots, n-1$ and $s$ with $l-1 / 2<s<n-1$ there exists $c_{n}=c_{n}(s)$, such that for all $a \geqq 1$ and all $\varepsilon>0$

$$
\begin{aligned}
& \left(b_{l}^{+}\right) \quad\left\|\rho_{a}^{s} R(\lambda+i \varepsilon ; V(g))^{l} P_{A}^{+} \rho_{a}^{l-s}\right\| \leqq c_{n} \lambda^{-l} \\
& \left(b_{l}^{-}\right) \quad\left\|\rho_{a}^{l-s} P_{A}^{-} R(\lambda+i \varepsilon ; V(g))^{l} \rho_{a}^{s}\right\| \leqq c_{n} \lambda^{-l}
\end{aligned}
$$

(ii) For $\eta>0, l-1 / 2<s<n-1$, the boundary values

and

$$
\rho_{a}^{s+\eta} R(\lambda+i 0 ; V(g)) P_{A}^{+} \rho_{a}^{l-s}
$$




$$
\rho_{a}^{l-s} P_{A}^{-} R(\lambda+i 0 ; V(g)) \rho_{a}^{s+\eta}
$$

exist in operator norm, are $l-1$ times continuously differentiable with Hölder continuous $l-1^{\text {st }}$ derivative which satisfies the estimates

$$
\begin{aligned}
& \left\|\left(\frac{d}{d \lambda}\right)^{l-1} \rho_{a}^{s+\eta} R(\lambda+i 0 ; V(g)) P_{A}^{+} \rho_{a}^{l-s}\right\| \leqq c_{n}(l-1) ! \lambda^{-l} \\
& \left\|\left(\frac{d}{d \lambda}\right)^{l-1} \rho_{a}^{l-s} P_{A}^{-} R(\lambda+i 0 ; V(g)) \rho_{a}^{s+\eta}\right\| \leqq c_{n}(l-1) ! \lambda^{-l} .
\end{aligned}
$$

We use the notation $(s)_{-}=\max \{-s, 0\}, s \in \boldsymbol{R}$.

Theorem 2.3. Let $V_{j}$ satisfy Assumption 1.1 for $n \geqq 3$. Then there exists $\lambda_{0}>0$ such that for all $\lambda \geqq \lambda_{0}$ and all $g \in \boldsymbol{R}_{+}^{N}$ satisfying (2.4) the following results hold.

(i) For $l=1, \cdots, n-2$ let $s_{1}, s_{2}$ satisfy $\left(s_{1}\right)_{-}+\left(s_{2}\right)_{-}<n-l$. There exists $c_{n}=$ $c_{n}\left(s_{1}, s_{2}\right)>0$ such that for $a \geqq 1$, and all $\varepsilon>0$

$$
\text { (c) }\left\|\rho_{a}^{s_{1}} P_{A}^{-} R(\lambda+i \varepsilon ; V(g))^{l} P_{A}^{+} \rho_{a}^{s_{2}}\right\| \leqq c_{n} \lambda^{-l} a^{-s_{1}-s_{2}}
$$

(ii) The boundary value

$$
\rho_{a}^{s_{1}} P_{A}^{-} R(\lambda+i 0 ; V(g)) P_{A}^{+} \rho_{a}^{s_{2}}
$$

exists in operator norm, is $l-1$ times continuously differentiable with an $l-1^{\text {st }}$ Hölder continuous derivative which satisfies the estimate

$$
\left\|\left(\frac{d}{d \lambda}\right)^{l-1} \rho_{a}^{s_{1}} P_{A}^{-} R(\lambda+i 0 ; V(g)) P_{A}^{+} \rho_{a}^{s_{2}}\right\| \leqq c_{n}(l-1) ! \lambda^{-l} a^{-s_{1}-s_{2}}
$$

\section{§3. Proofs}

We prove the results of Section 2. The first step is the following result.

Proposition 3.1. The general results $\left(a_{l}\right),\left(b_{l}^{ \pm}\right),\left(c_{l}\right)$ follow from these results for $l=1$.

Proof. This follows from a slightly modified version of [7, Lemma 2.1].

The next step consists in reducing the estimates to energy $\lambda=1$ by a scaling argument. The dilation group (scaling) on $L^{2}(X)$ is given by

$$
(U(\rho) f)(x)=\rho^{m / 2} f(\rho x) .
$$

Proposition 3.2. The results $\left(a_{1}\right),\left(b_{1}^{ \pm}\right),\left(c_{1}\right)$ follow from the estimates below. Let $V_{j}$ satisfy Assumption 1.1 for some $n \geqq 2$. Let

$$
W_{\lambda}(g)(x)=\sum_{j=1}^{N} g_{j} V_{j}\left(\lambda^{-1 / 2} \pi_{j}(x)\right)
$$


Then for $g \in \boldsymbol{R}_{+}^{N}$ with $\sum_{j=1}^{N} g_{j} \mu\left(V_{j}\right)<1 / \delta, \max \left\{g_{j}\right\} \leqq c_{0}$, with $c_{0}$ and $\delta>1$ from (2.4), we have the estimates below with $c$ independent of $\lambda>0$ :

$\left(\tilde{a}_{1}\right)$ For $s_{1}>1 / 2, s_{2}>1 / 2$ and all $\varepsilon>0, a \geqq 1$,

$$
\left\|\rho_{a}^{s_{1}} R\left(1+i \varepsilon ; W_{\lambda}(g)\right) \rho_{a}^{s_{2}}\right\| \leqq c a^{1-s_{1}-s_{2}}
$$

$\left(\tilde{b}_{1}^{ \pm}\right)$For $1 / 2<s<n$ and all $\varepsilon>0, a \geqq 1$,

$$
\left\|\rho_{a}^{s} R\left(1 \pm i \varepsilon ; W_{\lambda}(g)\right) P_{A}^{ \pm} \rho_{a}^{1-s}\right\| \leqq c
$$

$\left(\tilde{c}_{1}\right)$ For $s_{1}, s_{2}$ with $\left(s_{1}\right)_{-}+\left(s_{2}\right)_{-}<n-1$ and all $\varepsilon>0, a \geqq 1$,

$$
\left\|\rho_{a}^{s_{1}} P_{A}^{-} R\left(1+i \varepsilon ; W_{\lambda}(g)\right) P_{A}^{+} \rho_{a}^{s_{2}}\right\| \leqq c a^{-s_{1}-s_{2}}
$$

Proof. This result follows from the relation

$$
U(\rho)\left(H_{0}+V(g)-z\right)^{-1} U(\rho)^{-1}=\rho^{2}\left(H_{0}+\rho^{2} V(g)(\rho \cdot)-\rho^{2} z\right)^{-1}
$$

with $\rho=\lambda^{-1 / 2}, z=\lambda+i \varepsilon^{\prime}, W_{\lambda}\left(\lambda^{-1} g\right)(\cdot)=\rho^{2} V(g)(\rho \cdot)$, and the fact that $U(\rho)$ commutes with $A$.

To prove the estimates in Proposition 3.2 we verify the Mourre estimate. We have as quadratic forms on $\mathcal{S}\left(\boldsymbol{R}^{m}\right) \times \mathcal{S}\left(\boldsymbol{R}^{m}\right)$, where $\mathcal{S}\left(\boldsymbol{R}^{m}\right)$ denotes the Schwartz space :

$$
\begin{aligned}
i[H, A] & =2 H_{0}+i\left[W_{\lambda}(g), A\right] \\
& =2 H+i\left[W_{\lambda}(g), A\right]-2 W_{\lambda}(g) \\
& \leqq 2 H-2 \max \left\{0, \sum_{j=1}^{N} g_{j} \mu\left(V_{j}\right)\right\} \cdot I .
\end{aligned}
$$

We have used that $\mu\left(V_{j}\right)$ defined in (1.3) satisfies $\mu\left(V_{\jmath}(\rho \cdot)\right)=\mu\left(V_{\jmath}(\cdot)\right)$. Under the condition

$$
\sum_{\jmath=1}^{N} g_{\jmath} \mu\left(V_{\jmath}\right) \leqq \frac{1}{\delta}
$$

we get (see (2.4), remember that here $\lambda=1$ after scaling)

$$
i[H, A] \geqq 2 H-\frac{2}{\delta} I \text {. }
$$

Fix $\psi \in C_{0}^{\infty}(\boldsymbol{R}), \quad \psi \geqq 0, \quad \psi(t)=1$ for $3 / 4+1 / 4 \delta \leqq t \leqq 4 / 3$, supp $\phi \leqq[1 / 2+1 / 2 \delta, 3 / 2]$. Let $\Psi=\phi(H)$. It follows from (3.1) that the quadratic form $i[H, A]$ is closable. Let $i B_{1}$ denote the associated self-adjoint operator. We have $\mathscr{D}\left(B_{1}\right) \supseteqq \mathscr{D}\left(H_{0}\right)$. From (3.2) we conclude:

Lemma 3.3. We have

$$
\Psi_{i B_{1}} \Psi \geqq\left(1-\frac{1}{\delta}\right) \Psi^{2}
$$

for all $\lambda>0$ and all $g \in \boldsymbol{R}_{+}^{N}$ with $\sum_{\jmath=1}^{N^{*}} g_{\jmath} \mu\left(V_{\jmath}\right) \leqq 1 / \delta$. 
To prove the estimates $\left(\tilde{a}_{1}\right),\left(\tilde{b}_{1}^{ \pm}\right)$and $\left(\tilde{c}_{1}\right)$ in Proposition 3.2 we use the fact that the Mourre technique is constructive in the sense that constants in the estimates can be controlled explicitly, and parameter-dependence can be followed. In particular, since the constant $1-1 / \delta$ in the estimate (3.3) is uniform in $\lambda>0$ and $g \in \boldsymbol{R}_{+}^{N}$ satisfying $\sum_{j=1}^{N} g_{j} \mu\left(V_{j}\right) \leqq 1 / \delta$, we get the required uniformity in the estimates in Proposition 3.2. This follows from a careful examination of the proofs in $[11,14,10,7]$. For the estimate $\left(\tilde{a}_{1}\right)$, see also the computations in Section 6 and the paper [19], where closely related computations can be found. In the paper [8] similar computations are given for Stark effect Hamiltonians. For the estimates $\left(\tilde{b}_{1}^{ \pm}\right)$and $\left(\tilde{c}_{1}\right)$ in Proposition 3.2 one has to go through the details of the proof in [7]. These detailed computations are long, but straightforward, and will not be given here. We only note that in the proof of $\left(\tilde{b}_{1}^{ \pm}\right)$ the first step is to establish the estimate

$$
\left\|\rho_{a}^{s} R\left(1 \pm i \varepsilon ; W_{\lambda}(g)\right) P_{A}^{ \pm}\right\| \leqq c a^{1-s}
$$

for $s>1$ with $c$ uniform in $\lambda$ and $g$ as above.

\section{§4. Microlocal Resolvent Estimates}

In this section we prove resolvent estimates using localizations given by pseudodifferential operators. These results extend those obtained in [7] for the two-body case, and include high energy estimates. We shall give the proof of the first result in detail, and then sketch the proofs of the remaining results.

Let $w_{b}=\left(x^{2}+b^{2}\right)^{-1 / 2}$ for $b \geqq 1$. This weight function was used in the proofs in [1], but the dependence on $b$ was not made explicit, as in (4.3). The constant dependence for the estimate (4.3) was obtained in [19] in the case $l=1$.

The operator $H$ is bounded below. Choose $d_{0}>0$ such that for $d \geqq d_{0}$ we have $H+d \cdot I \geqq I$.

Lemma 4.1. Let $V_{j}$ satisfy Assumption 1.1 for $n=1$. Let $l \geqq 1$ be an integer. Let $b \geqq 1, \nu \geqq 1, a=\nu b, d \geqq d_{0}$ and $0 \leqq s \leqq l$. Then the operator $\rho_{a}^{-s}(H+d)^{-l} w_{b}^{s}$ is bounded and satisfies

$$
\left\|\rho_{a}^{-s}(H+d)^{-l} w_{b}^{s}\right\| \leqq c d^{-l}\left(d^{1 / 2}+\nu\right)^{s}
$$

where the constant $c$ is uniform in $\lambda$ and $g \in \boldsymbol{R}_{+}^{N}$ satisfying (2.4).

Proof. It suffices to prove the result for $s=0$ and $s=l$, and then use complex interpolation. For $s=0$ the estimate is obvious. For $s=l$ the result follows by commuting $x$ and $(H+d)^{-1}$. Due to the assumption on the coupling constant we have

$$
\left\|(-i \nabla)(H+d)^{-1}\right\| \leqq c d^{-1 / 2}
$$

for all $\lambda$ and all $g$ satisfying the conditions in the lemma. The rest now follows 
easily.

Theorem 4.2. Let $V_{j}$ satisfy Assumption 1.1 for $n \geqq 2$. Let $\lambda \geqq 1$ and $g \in \boldsymbol{R}_{+}^{N}$ satisfy (2.4). For $l=1, \cdots, n-1$ the following result holds: Let $s_{1}>l-1 / 2, s_{2}>$ $l-1 / 2$. Then there exists $c_{n}=c_{n}\left(s_{1}, s_{2}\right)$ such that for all $\varepsilon>0$ and $b \geqq 1$

$$
\left\|w_{b}^{s_{1}} R(\lambda+i \varepsilon ; V(g))^{l} w_{b}^{s_{2}}\right\| \leqq c_{n} \lambda^{-l / 2} b^{l-s_{1}-s_{2}} .
$$

Proof. Let $z=\lambda+i \varepsilon$. Write the resolvent as $R(z)$ to simplify the notation. Iterating the first resolvent equation

$$
R(z)=(z+d) R(-d) R(z)+R(-d)
$$

we obtain for any integer $k \geqq 1$

$$
R(z)=(z+d)^{k} R(-d)^{k} R(z)+\sum_{j=1}^{k}(z+d)^{\jmath-1} R(-d)^{j} .
$$

Let us first prove (4.3) for $l=1$. It suffices to consider $s_{1}=s_{2}=s, 1 / 2<s \leqq 1$, and prove the estimate for $\lambda \geqq d_{0}$. We write, using (4.4) for $k=2, d \geqq d_{0}$

$$
\begin{aligned}
w_{b}^{s} R(z) w_{b}^{s}= & \left(w_{b}^{s}(H+d)^{-1} \rho_{a}^{-s}\right)\left(\rho_{a}^{s} R(z) \rho_{a}^{s}\right)\left(\rho_{a}^{-s}(H+d)^{-1} w_{b}^{s}\right)(d+z)^{2} \\
& +w_{b}^{s}\left(R(-d)+(z+d) R(-d)^{2}\right) w_{b}^{s} .
\end{aligned}
$$

The first and third factor in the first summand are estimated using Lemma 4.1. The second factor is estimated using Theorem 2.1. We get

$$
\left\|w_{b}^{s} R(z) w_{b}^{s}\right\| \leqq d^{-2}\left(d^{1 / 2}+\nu\right)^{2 s} \lambda^{-1} a^{1-2 s}(d+|z|)^{2}+c b^{-2 s}\left(d^{-1}+d^{-2}(d+|z|)\right) .
$$

Note $a=\nu b$. Choose $\nu=\sqrt{\lambda}$ and $d=\lambda$ to get the required estimate. The general result is proved inductively, using the $l^{t h}$ power of (4.4), and estimating as above.

Corollary 4.3. Under the assumptions stated in Theorem 4.2 the boundary value

$$
w_{b}^{s_{1}} R(\lambda+i 0 ; V(g)) w_{b}^{s_{2}}
$$

exists in operator norm, and is $l-1$ times continuously differentiable with a Hölder continuous $l-1^{\text {st }}$ derivative which satisfies the estimate

$$
\left\|\left(\frac{d}{d \lambda}\right)^{l-1} w_{b}^{s_{1}} R(\lambda+i 0 ; V(g)) w_{b}^{s_{2}}\right\| \leqq c_{n}(l-1) ! \lambda^{-l / 2} b^{l-s_{1}-s_{2}} .
$$

Remark 4.4. Theorem 1.2 follows from Theorem 4.2 and Corollary 4.3.

Let $q_{ \pm} \in C^{\infty}\left(R^{2 m}\right)$ satisfy

$$
\left|\partial_{x}^{\alpha} \partial_{\xi}^{\beta} q_{ \pm}(x, \xi)\right| \leqq c_{\alpha \beta}\langle x\rangle^{-|\alpha|}\langle\xi\rangle^{-\mid \beta}
$$

for all multi-indices $\alpha$ and $\beta$, and furthermore 


$$
\operatorname{supp}\left(q_{ \pm}\right) \subseteq\{(x, \xi)| \pm x \cdot \xi \geqq \sigma| x|| \xi|,| \xi \mid \geqq v\}
$$

for some $\sigma, 0<\sigma<1$, and $v>0$. Let $f \in \mathcal{S}\left(R^{m}\right)$. Then $q_{ \pm}$define pseudodifferential operators $Q^{ \pm}$by

$$
\left(Q^{ \pm} f\right)(x)=(2 \pi)^{-m} \int e^{i x \cdot \xi} q_{ \pm}(x, \xi) \hat{f}(\xi) d \xi,
$$

where $\hat{f}$ denotes the Fourier transform. The operators $Q^{ \pm}$are bounded on $L^{2}(X)$. We obtain the following results :

Theorem 4.5. Let $V_{j}$ satisfy Assumption $1.1, n \geqq 2$. Let $\lambda \geqq 1$ and $g \in R_{+}^{N}$ satisfy (2.4). For $l=1, \cdots, n-1$ and s satisfying $l-1 / 2<s<n-1, b \geqq 1, \varepsilon>0$ there exists $c_{n}=c_{n}(s)>0$ such that

$$
\left\|w_{b}^{s} R(\lambda \pm i \varepsilon ; V(g))^{l} Q^{ \pm} w_{b}^{l-s}\right\| \leqq c_{n} \lambda^{-l / 2} .
$$

Theorem 4.6. Let $V_{j}$ satisfy Assumption 1.1, $n \geqq 3$. Let $\lambda \geqq 1$ and $g \in R_{+}^{N}$ satisfy (2.4). For $l=1, \cdots, n-2, s_{1}, s_{2}$ with $\left(s_{1}\right)_{-}+\left(s_{2}\right)_{-}<n-l, b \geqq 1, \varepsilon>0$ there exists $c_{n}=c_{n}\left(s_{1}, s_{2}\right)>0$ such that

$$
\left\|w_{b}^{s_{1}} Q^{-} R(\lambda+i \varepsilon ; V(g))^{l} Q^{+} w_{b}^{s_{2}}\right\| \leqq c_{n} \lambda^{-l / 2} b^{-s_{1}-s_{2}} .
$$

The proofs of Theorems 4.5 and 4.6 follow the line of those in [7], if one keeps track of the parameter dependence of the constants in the estimates. The details are omitted.

We conclude this section with an extension of Theorem 4.2 in the two-body case. Let $H=H_{0}+g V+\kappa U, g>0, \kappa>0$, where $V$ satisfies Assumption 1.1 for some $n_{0} \geqq 2$ on $X_{1}=X$, and $U$ satisfies for some $\beta>0$

$$
d_{n_{0}}(U)=\sup _{x \in X}\left((|x|+1)^{n_{0}-1+\beta}|U(x)|\right)<\infty .
$$

Theorem 4.7. Let $H$ satisfy the conditions above. There exists $\lambda_{0}>0$ such that for $\lambda \geqq \lambda_{0}, \lambda>\left(\kappa d_{n_{0}}(U) c_{n_{0}}\right)^{2}$ (the constant $c_{n_{0}}$ comes from Theorem 4.2 ), $\lambda \geqq$ $c_{0} g, \lambda \geqq \delta g \mu(V), \delta>1$, the following results hold. For $l=1, \cdots, n_{0}-1$ let $s_{1}>l$ $-1 / 2, s_{2}>l-1 / 2$. Then there exists a constant $\tilde{c}_{n_{0}}=\tilde{c}_{n_{0}}\left(s_{1}, s_{2}\right)>0$ such that for all $\varepsilon>0$

$$
\left\|w_{1}^{s_{1}}(H-\lambda-i \varepsilon)^{-l} w_{1}^{s_{2}}\right\| \leqq \tilde{c}_{n_{0}} \lambda^{-l / 2} .
$$

Proof. We have from Theorem 4.2 that

$$
\left\|w_{1}^{s}\left(H_{0}+g V-\lambda-i \varepsilon\right)^{-1} w_{1}^{s}\right\| \leqq c_{n_{0}} \lambda^{-1 / 2}, \quad s>1 / 2 .
$$

Thus we get

$$
\left\|w_{1}^{-s} \kappa U\left(H_{0}+g V-\lambda-i \varepsilon\right)^{-1} w_{1}^{s}\right\| \leqq c_{n_{0}} \lambda^{-1 / 2} \kappa d_{n_{0}}(U)
$$

for $l-1 / 2<s<n_{0}+\beta-3 / 2$. The conditions in the theorem on $\lambda$ ensure that the operator 


$$
I+\kappa U\left(H_{0}+g V-\lambda-i \varepsilon\right)^{-1}
$$

is invertible in the weighted $L^{2}$-space $L^{2, s}(X)$ with an inverse whose operator norm on that space is uniformly bounded in $\lambda$ satisfying the conditions above. We can now repeat the arguments in [9, Section 9] to get the theorem.

\section{§5. Semi-Classical Resolvent Estimates}

This section contains the semi-classical resolvent estimates that are easy consequences of our results in Section 2. We introduce $H(\hbar)=\hbar^{2} H_{0}+V(g), A(\hbar)$ $=\hbar A$ for $0<\hbar \leqq 1$. Note that $P_{A(h)}^{\leftarrow}=P_{A}^{ \pm}$.

Theorem 5.1. Replacing $H$ by $H(\hbar)$ and $A$ by $A(\hbar)$ in Theorems 2.1, 2.2, 2.3 introduces a factor $\hbar^{-1}$ on the right hand side of the estimates (2.5), (2.6), (2.7), (2.8), (2.9), (2.10). The constants on the right hand side of estimates (2.11) and (2.12) are independent of $\hbar$.

Proof. Using (2.5) we find for $H(\hbar)$ and $A(\hbar)$

$$
\begin{aligned}
& \left\|\left(A(\hbar)^{2}+a^{2}\right)^{-s_{1} / 2}(H(\hbar)-\lambda-i \varepsilon)^{-l}\left(A(\hbar)^{2}+a^{2}\right)^{-s_{2} / 2}\right\| \\
& =\hbar^{-s_{1}-s_{2}-2 l}\left\|\rho_{a}^{s_{1} / \hbar}\left(H_{0}+V\left(\hbar^{-2} g\right)-\hbar^{-2}(\lambda+i \varepsilon)\right)^{-l} \rho_{a / h}^{s_{2}}\right\| \\
& \leqq \hbar^{-s_{1}-s_{2}-2 l} c\left(\hbar^{-2} \lambda\right)^{-l}(a / \hbar)^{l-s_{1}-s_{2}} \\
& =c \lambda^{-l} a^{l-s_{1}-s_{2} \hbar^{-l}} .
\end{aligned}
$$

The other estimates are obtained in the same manner.

Theorem 5.2. Replacing $H$ by $H(\hbar)$ in Theorem 4.2, introduces a factor $\hbar^{-1}$ on the right hand side of the estimates (4.3), and (4.5).

Proof. This follows from Theorem 4.2 and Corollary 4.3 as in the proof of Theorem 5.1.

Remark 5.3. Semi-classical resolvent estimates similar to those in Theorem 5.2 have been obtained for two-body Schrödinger operators for the energy in a non-trapping energy interval in $[20,15,5]$. In [20] results on powers of the resolvent have also been obtained. Note that our estimate (2.11) is stronger than the result obtained in [20] because we use the sharp localizations $P_{A}^{ \pm}$.

\section{$\S 6$. Homogeneous Potentials}

In this section we give a result on the a priori resolvent estimate for $-\Delta+c|x|^{-\nu}$ on $L^{2}\left(\boldsymbol{R}^{m}\right)$ where $c \in \boldsymbol{R}$ and $0<\nu<\min \{m / 2,2\}$ with an explicit estimate for all constants. This result is given to show that such results are obtainable without too much additional effort, and to explain the method of 
proof in Section 3 by an example.

We use Mourre's differential inequality technique in the proof, but with a choice of auxiliary operators which is different from the one made by Mourre and in Section 3.

Theorem 6.1. Let $H=-\Delta+c|x|^{-\nu}, c \in \boldsymbol{R}, 0<\nu<\min \{m / 2,2\}$. The following estimate holds for all $\lambda>0, \varepsilon \neq 0$

$$
\begin{aligned}
& \left\|\left(1+x^{2}\right)^{-1 / 2}(H-\lambda-i \varepsilon)^{-1}\left(1+x^{2}\right)^{-1 / 2}\right\| \\
& \leqq \\
& \nu \sqrt{\lambda}\left[1+6 \sqrt{3} \sqrt{\nu /(2-\nu)}\left(1+2 \sqrt{\nu /(2-\nu)}+\frac{2+2 \nu+m}{\nu \sqrt{\lambda}}\right)^{1 / 2}\right. \\
& \left.\quad+\frac{2+2 \nu+m}{\nu \sqrt{\lambda}}\left(1+2 \sqrt{\nu /(2-\nu)}+\frac{2+2 \nu+m}{\nu \sqrt{\lambda}}\right)\left(\chi_{(1, \infty)}(\lambda)+4 \sqrt{\lambda} \chi_{(0,1)}(\lambda)\right)\right] .
\end{aligned}
$$

Proof. The proof will be given in some detail. Let $H_{0}=-\Delta$ and $V=c|x|^{-\nu}$ on $L^{2}\left(\boldsymbol{R}^{m}\right)$. As above, $A=1 / 2 i(x \cdot \nabla+\nabla \cdot x)$. With the restriction on $\nu$ in the theorem $V$ is a relatively compact operator perturbation of $H_{0}$, and thus $H=$ $H_{0}+V$ is self-adjoint with $\mathscr{D}(H)=\mathscr{D}\left(H_{0}\right)$. We have as quadratic forms on the Schwartz space:

Fix $\lambda>0$ and define

$$
\begin{aligned}
i[H, A] & =2 H_{0}+\nu V \\
& =(2-\nu) H_{0}+\nu H .
\end{aligned}
$$

$$
B^{*} B=(2-\nu) H_{0}+\nu \lambda,
$$

i. e. we replace $H$ by $\lambda$ in (6.2). Using [11, Proposition II.5] we find that the operator

$$
\left.G(\varepsilon)=H-\lambda-i \mu-i \varepsilon B^{*} B\right)^{-1}
$$

exists as a bounded operator for $\mu \varepsilon>0$. We consider only $\mu>0$ and $\varepsilon>0$ below. It satisfies

$$
\|G(\varepsilon)\| \leqq \frac{1}{\sqrt{\nu \lambda \varepsilon}}\|G(\varepsilon)\|^{1 / 2}
$$

and thus

$$
\|G(\varepsilon)\| \leqq \frac{1}{2 \lambda \varepsilon} .
$$

Let $w=\left(1+x^{2}\right)^{-1 / 2}$ to simplify the notation from section 4 , and define $F(\varepsilon)=$ $w G(\varepsilon) w$. We now derive the differential inequality. We use the relation

and find

$$
(H-\lambda) G(\varepsilon)=1+\left(i \mu+i \varepsilon B^{*} B\right) G(\varepsilon)
$$

$$
\begin{aligned}
\frac{d}{d \varepsilon} F(\varepsilon)= & i w G(\varepsilon)\left(\nu \lambda+(2-\nu) H_{0}\right) G(\varepsilon) w \\
= & i w G(\varepsilon) i\left[H-\lambda-i \mu-i \varepsilon B^{*} B, A\right] G(\varepsilon) w \\
& -i \nu w G(\varepsilon)(H-\lambda) G(\varepsilon) w+i \varepsilon 2(2-\nu) w G(\varepsilon) H_{0} G(\varepsilon) w
\end{aligned}
$$




$$
\begin{aligned}
= & w G(\boldsymbol{\varepsilon}) A w-w A G(\boldsymbol{\varepsilon}) w-i \nu w G(\boldsymbol{\varepsilon}) w \\
& +\nu w G(\boldsymbol{\varepsilon})\left(\boldsymbol{\mu}+\boldsymbol{\varepsilon} B^{*} B\right) G(\boldsymbol{\varepsilon}) w+i \varepsilon 2(2-\boldsymbol{\nu}) w G(\boldsymbol{\varepsilon}) H_{0} G(\boldsymbol{\varepsilon}) w .
\end{aligned}
$$

Mourre's quadratic estimate [11, Proposition II. 5] yields

$$
\left\|\sqrt{\mu+\varepsilon B^{*} B} G(\varepsilon) w\right\| \leqq\|w G(\varepsilon) w\|^{1 / 2} .
$$

Write $A=x \cdot(-i \nabla)-i m / 2$ and use $(2-\nu) H_{0} \leqq B^{*} B$ together with Mourre's quadratic estimate to get

$$
\|w A G(\boldsymbol{\varepsilon}) w\| \leqq \frac{m}{2}\|w G(\boldsymbol{\varepsilon}) w\|+\frac{1}{\sqrt{ } 2-\nu} \frac{1}{\sqrt{\varepsilon}}\|u G(\varepsilon) w\|^{1 / 2} .
$$

Finally we get the differential inequality

$$
\begin{gathered}
\left\|\frac{d}{d \varepsilon} F(\varepsilon)\right\| \leqq \frac{2}{\sqrt{2-\nu}} \frac{1}{\sqrt{\varepsilon}}\|F(\varepsilon)\|^{1 / 2}+(2+2 \nu+m)\|F(\varepsilon)\| \\
\|F(\varepsilon)\| \leqq \frac{1}{\nu \lambda \varepsilon} .
\end{gathered}
$$

This inequality is integrated in two iterations over the interval $(0, \sqrt{\lambda})$, if $0<$ $\lambda \leqq 1$, and over $(0,1 / \sqrt{\lambda})$, if $1 \leqq \lambda<\infty$ to obtain the existence of $F(0)$ and the bound in the theorem. We have also used the rough estimate

$$
-\log (s) \leqq s^{1-\delta} /(\delta-1), \quad 1<\delta<2, \quad 0<s \leqq 1
$$

and optimized the value of $\delta$ in the inequality.

The behavior in $\lambda$ for large and small $\lambda$ is optimal. Notice in particular that one obtains a decay rate $O\left(\lambda^{-1 / 2}\right)$ as $\lambda \rightarrow \infty$, even though the potential has a local singularity at zero.

The result above can be extended in several ways. We can obtain estimates for powers of the resolvent, and we can add a bounded potential with a decay rate $O\left(|x|^{-2}\right)$ as $|x| \rightarrow \infty$ and obtain the same resolvent estimates and decay in energy at infinity as above although getting explicit constants requires somewhat lengthy computations.

If one replaces the weight function $w$ with $\left(1+x^{2}\right)^{-s / 2}, s>1 / 2$, one has to use a number of iterations depending on the size of $s-1 / 2$. Thus one cannot obtain explicit constants in that case. One can however investigate the $\lambda$ dependence, and one finds analogous results.

The above result answers some questions in the paper [18]. Further results will be given elsewhere.

\section{§ 7. Remarks}

Remark 7.1. The technique developed here can also be applied to higher order operators. To compare with the results in $[1,13]$ we shall briefly com- 
ment on such results. Let $H_{0}=p(-i \nabla)$, where the symbol $p(\xi)=\sum_{|a|=m} a_{\alpha} \xi^{a}$ satisfies $p(\xi) \geqq c|\xi|^{m}$ for all $\xi \in \mathbb{R}^{m}$. We then have $i\left[H_{0}, A\right]=m H_{0}$, and we can repeat the arguments from the previous sections for operators $H=H_{0}+V$, where $V$ satisfies Assumption 1.1, or more generally, one can allow lower order terms in the perturbation. Under condition (2.4) the results in Theorems 2.1, 2.2 and 2.3 remain valid for this new operator. The estimates in Theorem 4.2 are changed, however. Lemma 4.1 requires a modification. The estimate (4.1) becomes

$$
\left\|\rho_{a}^{-s}(H+d)^{-l} w_{b}^{s}\right\| \leqq c d^{-l}\left(d^{1 / m}+\nu\right)^{s} .
$$

This comes from the changed estimate (4.2), which now reads

$$
\left\|(-i \nabla)(H+d)^{-1}\right\| \leqq c d^{-1 / m} \text {. }
$$

We therefore get resolvent estimates

$$
\left\|w_{b}^{s_{1}} R(\lambda+i \varepsilon ; V(g))^{l} w_{b}^{s_{2}}\right\| \leqq c_{n} \lambda^{-l(m-1) / m} b^{l-s_{1}-s_{2}} .
$$

This follows as in the proof of Theorem 4.2, where we now take $\nu=\lambda^{1 / m}$. The power of $\lambda$ for $l=1$ is the same as was found in $[1,13]$.

Remark 7.2. The results obtained in this paper can also be applied to some of the potentials considered in [17], for instance the potential

$$
V(x)=\frac{x_{1}}{\langle x\rangle},
$$

which satisfies the Assumption 1.1 for any $n$. Here we obtain the high energy estimates of Theorem 4.2 and the smoothness of the boundary values of the resolvent. In [17] a different method of proof based on a modified radiation condition is used for the case $l=1$.

\section{Acknowledgement}

Part of this research was supported by a grant from the US-NSF.

\section{References}

[1] Agmon, S., Spectral properties of Schrödinger operators and scattering theory, Ann. Scuola Norm. Sup. Pisa, (4) 2 (1975), 151-218.

[2] - Lectures on exponential decay of solutions of second order elliptic equations: Bounds on eigenfunctions of N-body Schrödinger operators, Princeton University Press, Princeton, (1982).

[3] Cycon, H. and Perry, P., Local time-decay of high energy scattering states for the Schrödinger equation, Math. Z. 188 (1984), 125-142.

[4] Froese, R. and Herbst, I., A new proof of the Mourre estimate, Duke Math. J. 49 (1982), 1075-1085.

[5] Gérard, C. and Martinez, A., Principe d'absorption limite pour des opérateurs de Schrödinger à longue portée, C.R. Acad. Sci. Paris, Ser. I, 306 (1988), 121-123. 
[6] Isozaki, H. and Kitada, H., Micro-local resolvent estimates for 2-body Schrödinger operators, J. Funct. Anal. 57 (1984), 270-300.

[7] Jensen, A., Propagation estimates for Schrödinger-type operators, Trans. Amer. Math. Soc. 291 (1985), 129-144.

[8] —, Precise resolvent estimates for Stark effect Hamiltonians, In: Proceedings of the International Symposium on Partial Differential Equations, April 1988, Holzhau, GDR, Teubner-texte, to appear.

[9] Jensen, A. and Kato, T., Spectral properties of Schrödinger operators and time decay of the wave functions, Duke Math. J. 46 (1979), 583-611.

[10] Jensen, A., Mourre, E. and Perry, P., Multiple commutator estimates and resolvent smoothness in quantum scattering theory, Ann. Inst. H. Poincaré, Sect. A (N.S) 41 (1984), 207-225.

[11] Mourre, E., Absence of singular continuous spectrum for certain self-adjoint operators, Commun. Math. Phys 78 (1981), 391-408.

[12] Murata, M., High energy resolvent estimates, I, first order operators, J. Math. Soc. Japan 35 (1983), 711-733.

[13] High energy resolvent estimates, II, higher order elliptic operators, $J$. Math. Soc. Japan 36 (1984), 1-10.

[14] Perry, P., Sigal, I. and Simon, B., Spectral analysis of N-body Schrödinger operators, Ann. of Math. (2) 114 (1981), 519-567.

[15] Robert, D. and Tamura, H., Semi-classical estimates for resolvents and asymptotics for total scattering cross-section, Ann. Inst. H. Poincaré, Phys. théor., 46 (1987), $415-442$.

[16] Saito, Y., The principle of limiting absorption for the non-selfadjoint Schrödinger operator in $\boldsymbol{R}^{N},(N \neq 2)$, Publ. RIMS Kyoto Univ. 9 (1974), 397-428.

[17] - Schrödinger operators with a nonspherical radiation condition, Pacific J. Math. 126 (1987), 331-359.

[18] Yafaev, D.R., Spectral properties of the Schrödinger operator with a potential having a slow falloff, Funct. Anal Appl. 16 (1982), 280-286.

[19] - The Eikonal approximation and the asymptotics of the total scattering cross-section for the Schrödinger equation, Ann. Inst. H. Poincaré, Phys. théor., 44 (1986), 397-425.

[20] Wang, X.P., Time-decay of scattering solutions and resolvent estimates for semiclassical Schrödinger operators, J. Diff. Eq. 71 (1988), 348-395. 
